\title{
Dopamine release in the nucleus accumbens during sexual behavior in prenatally stressed adult male rats
}

\author{
Chih-Tien Wang, Ruey-Ling Huang, Mei-Yun Tai, Yuan-Feen Tsai*, Ming-Tsung Peng \\ Department of Physiology, College of Medicine, National Taiwan University, I Jen-Ai Road, 1st Section, Taipei, Taiwan (10018), ROC
}

Received 22 August 1995; revised version received 25 September 1995; accepted 26 September 1995

\begin{abstract}
In vivo microdialysis experiments were performed on the nucleus accumbens (NAc) during observation of sexual behavior (including motivation and copulation) to determine if there were any changes in NAc dopamine (DA) transmission in prenatally stressed (PS) adult male rats. Approximate 37\% of control males and 83\% of PS males did not exhibit copulation during the sexual behavior tests and no significant changes in NAc DA release were seen during exposure to estrous females. In contrast, both control and PS males that displayed copulatory behavior showed a marked increase in NAc DA release when presented with a sexually receptive female behind a screen and this increased further during actual copulation. The increase in DA release in copulatory PS males was not significantly different from that in sexually active control males. In addition, a similar extent in DA release induced by high potassium perfusate was observed in all rats. These results suggest that prenatal stress may result in a deficit in DA neurotransmission in the NAc and this deficit may possibly cause impaired male sexual behavior in rats.
\end{abstract}

Keywords: Microdialysis; Prenatal stress; Nucleus accumbens; Dopamine; Sexual motivation; Copulation

Demasculinized copulatory behavior has been reported in prenatally stressed (PS) adult male rats, with only $26 \%$ displaying copulation during exposure to sexually receptive female rats [16]. Further studies indicate that the decreased copulatory behavior seen in PS male rats is not due to impaired locomotor activity [10] or to the influence of abnormal maternal behavior of their stressed mothers [5]. In addition, a deficiency in sexual motivation to estrous females is evident in PS males [7]. Numerous studies have supported the view that sexual motivation and copulation in male rats are associated with dopamine (DA) release in the nucleus accumbens (NAc) $[2,3,9,13-$ 15]. The significant increase in DA release, seen in the NAc of sexually active male rats exposed to receptive females, is not due to novelty, locomotor activity or former sexual experiences, but to sexual arousal and copulation $[2,17]$. Thus, the purpose of the present study was to investigate by means of in vivo microdialysis whether the decreased male sexual motivation and copulation ob-

* Corresponding author. Tel.: +88623970800 , extn. 8240 ; fax: +886 23964350 . served in PS rats correlates with abnormal DA neurotransmission in the NAc.

Adult female Wistar rats were housed individually under a reversed $14: 10 \mathrm{~h}$ light-dark cycle (lights on at $2000 \mathrm{~h}$ ) at $22 \pm 1{ }^{\circ} \mathrm{C}$, with food and water available ad libitum. From day 15 to day 19 of gestation, the pregnant animals in the PS group $(n=10)$ were immobilized in a $5 \times 5 \times 11 \mathrm{~cm}$ metal cage for $2 \mathrm{~h}$ per day. The females in the control group $(n=12)$ were left undisturbed for the whole pregnancy period, except for routine maintenance. After weaning, the male pups were housed in groups of three or four and, from 3 to 6 months of age, were tested ten times for copulation with receptive females. Approximately $37 \%(14 / 38)$ of control males and $83 \%$ (24/29) of PS males displayed no copulatory behavior. Based on these results, male rats were subdivided into four groups for microdialysis studies: (1) control copulators (control-C, $n=6$ ); (2) control non-copulators (control-NC, $n=7$ ); (3) PS copulators (PS-C, $n=4$ ) and (4) PS non-copulators (PS-NC, $n=7$ ).

All male rats were anesthetized with ketamine $(45 \mathrm{mg} /$ $\mathrm{kg}$, i.p.) and sodium pentobarbital $(10 \mathrm{mg} / \mathrm{kg}$, i.p. $)$. A home-made microdialysis probe (length of dialysis sur- 
face, $2 \mathrm{~mm}$ ), mainly constructed as described by Lee et al. [8], was stereotaxically implanted into the NAc (coordinates of probe tip relative to bregma were: $\mathrm{AP},+1.8 \mathrm{~mm}$; DV , $-8.2 \mathrm{~mm}$; ML, $+1.4 \mathrm{~mm}$ ) [12] and then secured with dental cement. Recovery rates (mean \pm SEM) were $12.13 \pm 0.62 \%$ for DA, $11.67 \pm 0.55 \%$ for 3,4-dihydroxyphenylacetic acid (DOPAC) and $11.14 \pm 0.69 \%$ for homovanillic acid (HVA). During surgery, the microdialysis probe was perfused continuously with artificial cerebrospinal fluid (ACSF) $(140 \mathrm{mM} \mathrm{NaCl}, 3.0 \mathrm{mM} \mathrm{KCl}$, $1.0 \mathrm{mM} \mathrm{MgCl}, 1.2 \mathrm{mM} \mathrm{CaCl} 2$ and $0.04 \mathrm{mM}$ ascorbic acid) at a rate of $1 \mu \mathrm{l} / \mathrm{min}$. Following surgery, the perfusion rate was decreased to $0.3 \mu \mathrm{l} / \mathrm{min}$ and the experimental rats housed individually in transparent Plexiglas bowlcages.

The male sexual behavior test, with concurrent microdialysis sampling, was performed 1 day after surgery during the rats' dark phase. Ninety minutes before dialysate sampling, the perfusion rate was returned to $1 \mu \mathrm{l} / \mathrm{min}$ and microdialysis samples were collected every 20 min throughout the experiment. Twenty minutes prior to sampling, the experimental rat was transferred to a transparent Perspex mating chamber $(45 \times 35 \times 35 \mathrm{~cm})$, containing a vertical wire-mesh screen $(30 \times 30 \mathrm{~cm})$ that divided the chamber into two compartments (compartment $\mathrm{A}, 30 \times 35 \times 35 \mathrm{~cm}$ and compartment $\mathrm{B}, 15 \times$ $35 \times 35 \mathrm{~cm}$ ). The behavioral test consisted of four consecutive phases as follows: (a) phase I (basal phase, $60 \mathrm{~min}$ ), the male rat was kept in compartment $\mathrm{A}$; (b) phase II (motivational phase, $40 \mathrm{~min}$ ), a receptive estrous female was placed behind the screen in compartment B; (c) phase III (copulatory phase, $40 \mathrm{~min}$ ), the wire-mesh screen was removed to allow physically sexual interaction; and (d) phase IV (postcopulatory phase, $80 \mathrm{~min}$ ), the female was removed. During phase III, the number of mounts, intromissions and ejaculations and their latencies were recorded.

When the behavioral test was complete, the perfusate was changed to a high potassium ACSF $(43 \mathrm{mM} \mathrm{NaCl}$, $100 \mathrm{mM} \mathrm{KCl}, 1.0 \mathrm{mM} \mathrm{MgCl}_{2}, 1.2 \mathrm{mM} \mathrm{CaCl}_{2}$ and $0.04 \mathrm{mM}$ ascorbic acid) for $20 \mathrm{~min}$, and dialysis samples collected for a further $40 \mathrm{~min}$ period. Histological sectioning of the brain confirmed the accuracy of probe placement; only data from those experiments in which the probe located in the NAc were analyzed. Microdialysis samples were analyzed by high performance liquid chromatography with electrochemical detection and a mobile phase delivered at a flow rate of $50 \mu \mathrm{l} / \mathrm{min}$ onto a microbore column.

During phase I, the basal concentrations (mean \pm SEM; values corrected for individual probe recoveries) of DA, DOPAC and HVA were: $4.39 \pm 0.75,691.0 \pm 168.6$ and $402.7 \pm 76.1 \mathrm{pg} / \mu 1$ in the control-C group; $3.88 \pm$ $0.54,607.8 \pm 231.5$ and $383.0 \pm 55.5 \mathrm{pg} / \mu \mathrm{l}$ in the con trol-NC group; $3.13 \pm 0.96,692.2 \pm 139.5$ and $473.8 \pm$ $120.3 \mathrm{pg} / \mu 1$ in the PS-C group; and $4.69 \pm 0.89,845.4 \pm$

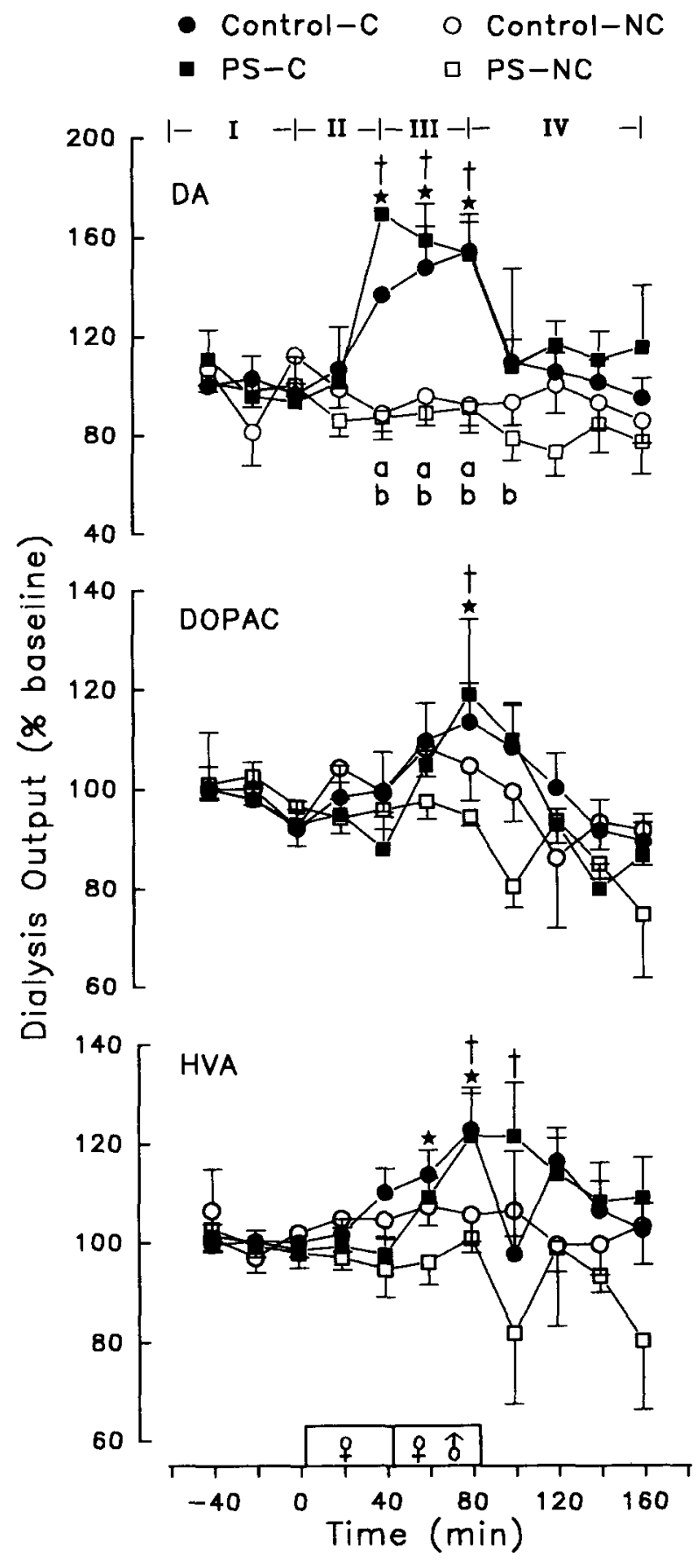

Fig. 1. Temporal changes in dialysate concentrations of DA, DOPAC, and HVA in the NAc of male rats during the sexual behavior test. Repeated measures ANOVAs were performed on the percentage change in DA, DOPAC, and HVA obtained in six dialysis samples (the first and the last value from phase I and IV, respectively; two values from each of phases II and III). The significance levels was $P<0.05$ for each of the following comparisons: a, control-C versus control-NC; b, PS-C

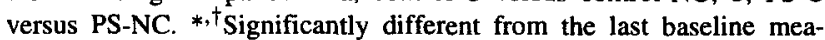
surement in control-C and PS-C, respectively (Scheffé's method). Roman numerals indicate four consecutive phases of the behavioral test. 

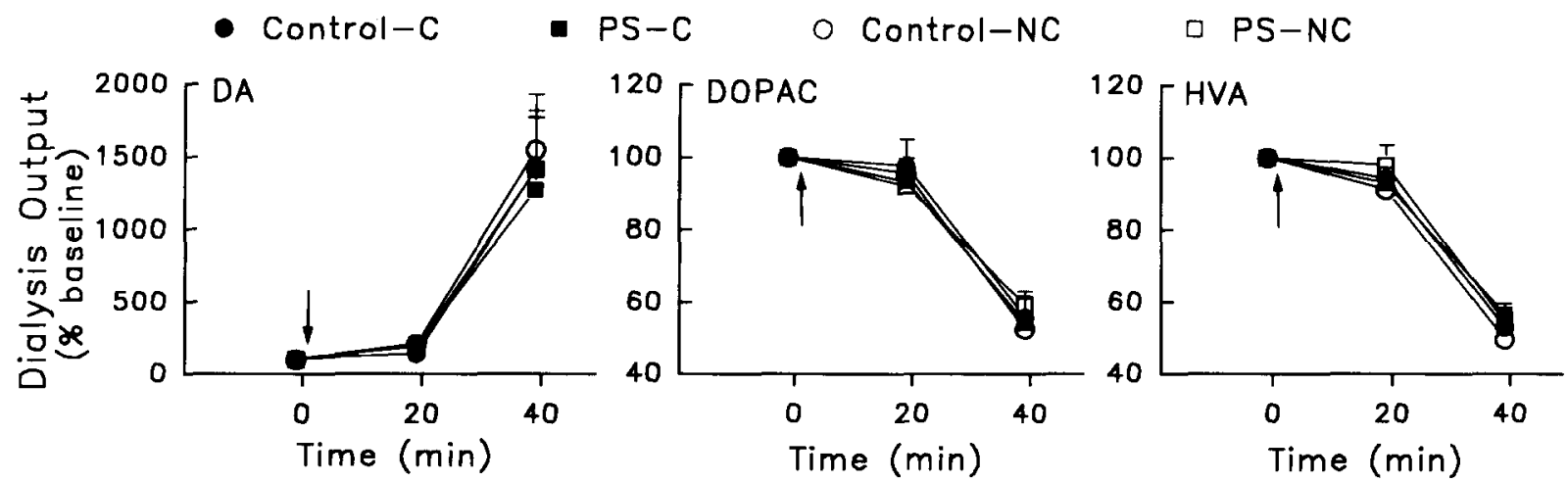

Fig. 2. Time course in male rats of changes in extracellular levels of DA and its metabolites in the NAc induced by a high potassium challenge for $20 \mathrm{~min}$. Arrows represent the start of high potassium perfusion. Repeated measures ANOVAs were conducted on percentage change in DA, DOPAC and HVA in three dialysis samples (pre-infusion value designated as $100 \%$ ). No significant difference in levels of DA, DOPAC or HVA was seen between the four groups.

81.2 and $445.3 \pm 49.2 \mathrm{pg} / \mu 1$ in the PS-NC group, respectively. There was no significant difference in basal values between the groups. During the sexual behavior observation, ANOVA tests showed a significant overall difference for changes in DA output between copulator and non-copulator groups $(F(1,20)=24.86, P<0.001)$, a significant time effect $(F(5,100)=6.66, P<0.001)$ and a significant interaction between group and time $(F(5,100)=12.20, \quad P<0.001)$. Subsequent post-hoc analysis showed that there was a significant increase in NAc extracellular DA content in both control-C and PS-C males during the second part of phase II and all of phase III. The increase in accumbens DA release during phases II and III was not significantly different between control$\mathrm{C}$ and PS-C rats. In contrast, for both control-NC and PS$\mathrm{NC}$ males, there were no statistically significant changes in accumbens extracellular DA levels during the entire sexual behavioral test (Fig. 1). During phase III, both control-C and PS-C males exhibited copulatory behaviors, while groups of control-NC and PS-NC rats still showed no copulatory activity. No statistically significant difference in copulatory performance was seen between control-C and PS-C males. These results indicate that most PS males display no copulation and exhibit a deficit in NAc DA release.

Acidic metabolites of DA increased in both control$\mathrm{C}$ and PS-C rats during the sexual behavioral test (Fig. 1). ANOVAs revealed a significant time effect for both DOPAC $(F(5,100)=7.21, P<0.001)$ and HVA $(F(5,100)=7.21, P<0.001)$. Post-hoc analysis showed that there was a $20-40 \mathrm{~min}$ delay in the increase in DOPAC and HVA output compared with that for DA output. These results are consistent with previous reports $[14,15]$.

In order to determine whether the presynaptic DA content was diminished in the NAc of PS males, high potassium perfusate, which elicits the DA release [6], was administrated after the sexual behavior test. As shown in Fig. 2, a significant increase in NAc extracellular DA levels was seen $40 \mathrm{~min}$ after high potassium challenge in all four groups $(F(2,34)=30.82, P<0.001)$, while a marked decrease in DA metabolites was also detected in all four groups $(F(2,34)=81.92, P<0.001$ for DOPAC; $F(2,34)=92.50, P<0.001$ for HVA $)$. No significant difference in extracellular levels of DA and its metabolites was found in the four groups after high potassium treatment. These data suggest that prenatal stress does not affect the presynaptic DA content in the NAc, although most PS male rats exhibit a deficit in NAc DA transmission.

The main findings of the present study are that most PS male rats (PS-NC) and approximate $37 \%$ of control males (control-NC) showed no copulatory behaviors or any changes in NAc extracellular levels of DA, DOPAC and HVA during exposure to receptive females, but only few PS males (PS-C) did show marked increases in release of DA and its metabolites in the NAc during motivational and copulatory phase of the sexual behavior test; these increases were not significantly different from those seen in control-C males. These data therefore suggest that exposing pregnant rats to intense environmental stressors may impair NAc DA release mechanisms in their male offspring, and the NAc DA transmission may play a crucial role in both male sexual motivation and copulation in rats. However, it should be noted that approximately $37 \%$ of control males in the present study failed to display copulatory behavior. The reasons for this phenomenon remain unclear.

Since electrophysiological, neurochemical and behavioral evidence has demonstrated that functions of dopaminergic neurons in the NAc are regulated by glutamate transmission $[1,4,11]$, the deficit in the NAc DA release in non-copulatory males might be caused by dysfunction of glutamatergic system. Further work is therefore needed to clarify the interactions between dopaminergic and glutamatergic systems within the NAc in these rats.

In summary, these results suggest that prenatal stress leads to a deficit in NAc DA transmission in male rats 
which show no changes in DA release during exposure to sexually receptive fermales. These data should provide important information on the underlying mechanism of decreased male sexual behavior in PS rats.

This work was supported by the National Science Council Grant NSC-84-2331-B002-294 and NSC-842331-B002-296, ROC.

[1] Burns, L.H., Everitt, B.J., Kelley, A.E. and Robbins, T.W., Glutamate-dopamine interactions in the ventral striatum: role in locomotor activity and responding with conditioned reinforcement, Psychopharmacology, 115 (1994) 516-528.

[2] Damsma, G., Pfaus, J.G., Wenkstern, D., Phillips, A.G. and Fibiger, H.C., Sexual behavior increases dopamine transmission in the nucleus accumbens and striatum of male rats: comparison with novelty and locomotion, Behav. Neurosci., 106 (1992) 181-191.

[3] Fumero, B., Fernandez-Vera, J.R., Gonzalez-Mora, J.L. and Mas, M., Changes in monoamine turnover in forebrain areas associated with masculine sexual behavior: a microdialysis study, Brain Res., 662 (1994) 233-239.

[4] Hamilton, M.H., De Belleroche, J.S., Gardiner, I.M. and Herber, L.J., Stimulatory effect of $\mathrm{N}$-methyl aspartate on locomotor activity and transmitter release from rat nucleus accumbens, Pharmacol. Biochem. Behav., 25 (1986) 943-948.

[5] Herrenkohl, L.R. and Whitey, J.B., Effects of prepartal stress on postpartal nursing behavior, litter development and adult sexual behavior, Physiol. Behav., 17 (1976) 1019-1021.

[6] Kihara, T., Ikeda, M., Miyazaki, H. and Matsushita, A., Influence of potassium concentration in microdialysis perfusate on basal and stimulated striatal dopamine release: effect of ceruletide, a cholecystokinin-related peptide, J. Neurochem., 61 (1993) 1859 1864.

[7] Kinsley, C.H., Mann, P.E. and Bridges, R.S., Diminished luteiniz- ing hormone release in prenatally stressed male rats after exposure to sexually receptive females, Physiol. Behav., 52 (1992) 925928.

[8] Lee, E.H.Y., Chang, S.Y. and Chen, A.Y.J., CRF facilitates NE release from the hippocampus: a microdialysis study, Neurosci. Res., 19 (1994) 327-330.

[9] Mas, M., Gonzalez-Mora, J.L., Louilot, A., Solé, C. and Guadalupe, $T$., Increased dopamine release in the nucleus accumbens of copulating male rats as evidenced by in vivo voltammetry, Neurosci. Lett., 110 (1990) 303-308.

[10] Masterpasqua, F., Chapman, R.H. and Lore, R.K., The effects of prenatal psychological stress on the sexual behavior and reactivity of male rats, Dev. Psychobiol., 9 (1976) 403-411.

[11] Murase, S., Grenhoff, J., Chouvet, G., Gonon, F.G. and Svensson, T.H., Prefrontal cortex regulates burst firing and transmitter release in rat mesolimbic dopamine neurons studied in vivo, Neurosci. Lett., 157 (1993) 53-56.

[12] Paxinos, G. and Watson, C., The Rat Brain in Stereotaxic Coordinates, 2nd Edn., Academic Press, Orlando, 1986

[13] Pfaus, J.G. and Phillips, A.G., Role of dopamine in anticipatory and consummatory aspects of sexual behavior in the male rat, Behav. Neurosci., 105 (1991) 727-743.

[14] Pfaus, J.G., Damsma, G., Nomikos, G.G., Wenkstern, D.G., Blaha, C.D., Phillips, A.G. and Fibiger, H.C., Sexual behavior enhances central dopamine transmission in the male rat, Brain Res., 530 (1990) 345-348.

[15] Pleim, E.T., Matochik, J.A., Barfield, R.J. and Auerbach, S.B., Correlation of dopamine release in the nucleus accumbens with masculine sexual behavior in rats, Brain Res., 524 (1990) 160163.

[16] Ward, I.L., Prenatal stress feminizes and demasculinizes the behavior of males, Science, 175 (1972) 82-84.

[17] Wenkstern, D., Pfaus, J.G. and Fibiger, H.C., Dopamine transmission increases in the nucleus accumbens of male rats during their first exposure to sexually receptive female rats, Brain Res., 618 (1993) 41-46. 